Corrigendum

\title{
Corrigendum to "Comparison of the Efficacy of Three Loading Doses of Intravitreal Injection of Conbercept with Injection Combined with PDT for the Treatment of PCV"
}

\author{
Fengjiao Li, ${ }^{1,2}$ Aihua Ma $\mathbb{D}^{3,4}$ and Bojun Zhao $\mathbb{D i D}^{1,2}$ \\ ${ }^{1}$ Department of Ophthalmology, Shandong Provincial Hospital Affiliated to Shandong First Medical University, Jinan, \\ Shandong 250021, China \\ ${ }^{2}$ Department of Ophthalmology, Shandong Provincial Hospital, Cheeloo College of Medicine, Shandong University, Jinan, \\ Shandong 250021, China \\ ${ }^{3}$ Department of Pediatrics, Shandong Provincial Hospital Affiliated to Shandong First Medical University, Jinan, \\ Shandong 250021, China \\ ${ }^{4}$ Department of Pediatrics, Shandong Provincial Hospital, Cheeloo College of Medicine, Shandong University, Jinan, \\ Shandong 250021, China
}

Correspondence should be addressed to Aihua Ma; aihuama@hotmail.co.uk and Bojun Zhao; zhaobojun0708@163.com Received 22 June 2020; Accepted 23 June 2020; Published 30 August 2020

Copyright (c) 2020 Fengiiao Li et al. This is an open access article distributed under the Creative Commons Attribution License, which permits unrestricted use, distribution, and reproduction in any medium, provided the original work is properly cited.

In the article titled "Comparison of the Efficacy of Three Loading Doses of Intravitreal Injection of Conbercept with Injection Combined with PDT for the Treatment of PCV" [1], authors Dr. Fengiiao Li and Dr. Bojun Zhao were not affiliated to "Department of Ophthalmology, Shandong Provincial Hospital, Cheeloo College of Medicine, Shandong University, Jinan, Shandong, 250021, China," and Dr. Aihua Ma was not affiliated to "Department of Pediatrics, Shandong Provincial Hospital, Cheeloo College of Medicine, Shandong University, Jinan, Shandong, 250021, China."

The corrected list of affiliations is shown in the author information above.

\section{References}

[1] F. Li, A. Ma, and B. Zhao, "Comparison of the efficacy of three loading doses of intravitreal injection of conbercept with injection combined with PDT for the treatment of PCV," BioMed Research International, vol. 2020, Article ID 2428348, 5 pages, 2020. 